\title{
Application of High-Resolution MALDI-TOFMS with a Spiral Ion Trajectory for the Structural Characterization of Free Radical Polymerized Methacrylate Ester Copolymers
}

\author{
Hiroaki Sato, ${ }^{* 1}$ Yoko Ishii, ${ }^{1}$ Hikaru Momose, ${ }^{2}$ Takafumi Sato, ${ }^{3}$ and Kanae Teramoto ${ }^{4}$ \\ Research Institute for Environmental Management Technology, National Institute of \\ Advanced Industrial Science and Technology (AIST), Tsukuba, Japan \\ ${ }^{2}$ Corporate Research Laboratories, Mitsubishi Rayon Co., Ltd., Otake, Japan \\ ${ }^{3}$ MS Business Unit, JEOL Ltd., Akishima, Japan \\ ${ }^{4}$ Advanced Technology Department, JEOL Ltd., Akishima, Japan
}

\begin{abstract}
The structural characterization of copolymers by matrix-assisted laser desorption/ionization time-of-flight mass spectrometry (MALDI-TOFMS) remains a challenging task, since their random comonomer distribution creates very complicated mass spectra. In this study, a high-resolution TOF mass spectrometer with a spiral ion trajectory was applied to the structural and compositional characterization of free radical copolymerized poly(methyl methacrylate-co-tert-butyl methacrylate), poly(MMA-co-tBMA)s in ethyl lactate acting as a chain transfer agent. Virtually complete peak assignments of the isobaric components within the poly(MMA-co-tBMA)s served to identify the end-group combinations and copolymer compositions of individual copolymer components, allowing the distributions of comonomer compositions and six types of end-group combinations to be evaluated.
\end{abstract}

Keywords: MALDI spiral-TOFMS, copolymer characterization, chemical composition, end group structure

(Received October 29, 2012; Accepted December 10, 2012)

\section{INTRODUCTION}

Polymer materials used in advanced products, including electrical and optical devices, generally consist of copolymers. For reason of its low cost, most industrial copolymers have been produced using free radical polymerization. Improvement of copolymer materials has been accomplished by detailed characterization of copolymers, including copolymer composition and comonomer sequences, as well as end-group structures, molecular weights, and their distributions.

Matrix-assisted laser desorption/ionization time-of-flight mass spectrometry (MALDI-TOFMS) is commonly used as a technique for polymer characterization. ${ }^{1)}$ Copolymer characterization has also been attempted using MALDITOFMS. $^{2-8)}$ However, the structural characterization of copolymers remains a challenging task, since the random nature of comonomer distribution generates very complicated mass spectra. During free radical polymerization of copolymers, a variety of end-group combinations are formed, resulting in extremely intricate mass spectra. A mass of different combinations of comonomer distributions and end-groups increases the likelihood of isobaric interference, in which the peaks of different chemical compositions

\footnotetext{
* Correspondence to: Hiroaki Sato, Research Institute for Environmental Management Technology, National Institute of Advanced Industrial Science and Technology (AIST), Tsukuba 305-8569, Japan, e-mail: sato-hiroaki@ aist.go.jp

This manuscript was submitted to the Special Issue: Advanced Techniques of MALDI-TOF MS in Ionization, Instrumentation and Applications, Vol. 1 (2012), No. 1
}

with the same nominal mass overlap. For these reasons, the characterization of copolymers by MALDI-TOFMS currently appears to be limited to copolymers with a uniform end-group structure.

Peak separation of isobaric components within complicated copolymers requires high-resolution mass spectrometry. Fourier transform ion cyclotron mass spectrometry (FTICR-MS) is the most powerful tool for separating isobaric components at high resolution and readily determines the chemical composition of copolymers. Several applications of MALDI-FTICR-MS have been reported for the characterization of copolymers. ${ }^{9-12)}$ However, special skills for operation and careful maintenance of FTICR-MS are required, especially in the case of combination with a MALDI source.

A new type of TOFMS instrument with a spiral ion trajectory (spiral-TOFMS) has been developed. ${ }^{13,14)}$ The flight path of the ions along the spiral ion trajectory is approximately $17 \mathrm{~m}(2.1 \mathrm{~m} \times 8$ turns), which reveals a high massresolving power of 80,000 at full width at half maximum (FWHM) for $m / z$ ca. 2,564 of a peptide sample. ${ }^{14)}$ This resolving power should in many cases be sufficient to separate the peaks of isobaric components within copolymers. The advantage of spiral-TOFMS over FTICR-MS is that high-resolution mass spectrometry can be readily performed using basically the same procedure as with conventional TOFMS instruments.

In this study, a detailed structural characterization of copolymers was attempted using MALDI spiral-TOFMS. The model copolymers were prepared by free radical copolymerization of methyl methacrylate (MMA), with tertbutyl methacrylate (tBMA) in ethyl lactate (EL) acting as the chain transfer agent. MALDI spiral-TOFMS appears to have 
the potential to perform almost complete peak assignments of isobaric components observed in high-resolution mass spectra. Detailed structural characterization (end-group structures and their combinations, copolymer compositions and their distributions) of poly(MMA-co-tBMA)s with different copolymer compositions is demonstrated in this study.

\section{EXPERIMENTAL}

\section{Polymer samples}

Table 1 summarizes the properties of the poly(MMAco-tBMA)s used in this study. Copolymer samples were prepared as follows. A mixture of monomer (20 wt $\%)$ and 2,2-azobis(2,4-dimethylvaleronitrile) (AVN) (5 mol\% of the monomer mixture) was dissolved in ethyl lactate. Polymerization was carried out at $80^{\circ} \mathrm{C}$ under a nitrogen atmosphere. After $4 \mathrm{~h}$, the polymerization mixture was cooled to room temperature and poured into a large volume of methanol-water mixture $(4: 6 \mathrm{vol} / \mathrm{vol})$. The polymer precipitated and was collected by filtration, then dried overnight at $60^{\circ} \mathrm{C}$ in vacuo. The copolymer composition of each sample was determined from the integral intensities of the ${ }^{1} \mathrm{H}$ NMR signals of the ester substituents of MMA and tBMA units on a JEOL ECX400 spectrometer.

\section{MALDI spiral-TOFMS}

As the matrix for sample ionization, 2-[(2E)-3-(4-tertbutylphenyl)-2-methyl-2-prop-2-enylidene]malononitrile, called DCTB matrix, ${ }^{15-17)}$ which provides clear MALDI spectra at lower laser power, was employed. About $10 \mathrm{mg}$ of DCTB and about $1 \mathrm{mg}$ of polymer sample were dissolved in tetrahydrofuran (THF). About $1 \mu \mathrm{L}$ of $\mathrm{NaI}$ solution $(1 \mathrm{mg} /$ $\mathrm{mL}$ in acetone) was precoated on the MALDI target plate. Next, approximately $2 \mu \mathrm{L}$ of the sample/matrix $(1 / 10 \mathrm{v} / \mathrm{v})$ mixture was pippetted onto the plate, which was then dried in air. MALDI mass spectra were observed using a SpiralTOFMS (JEOL, Tokyo, Japan). The details of the instrument's configuration are described in ref. 14). In brief, ions generated by irradiation with a nitrogen laser (MNL103LD; Lasertechnik Berlin, Berlin, Germany; $\lambda=337 \mathrm{~nm}$ ) were accelerated at $20 \mathrm{kV}$. The ions then passed along a spiral ion trajectory, with a flight length of approximately $17 \mathrm{~m}$, during which they were separated according to their massto-charge ratio. The settings of delay time and grid voltage were optimized to maintain the FWHM constant at $\Delta M=$ $0.02-0.03 \mathrm{Da}$ over the range of $m / z 600-3,000$. Mass calibration was made with a poly(methyl methacrylate) (PMMA) standard (peak-top molecular weight, $M_{\mathrm{p}}=1,310$ ) purchased from Polymer Laboratories (Church Stretton, UK). Three mass spectra for each sample were collected. The mass spectral data analysis was supported by Polymerix software
(Sierra Analytics, Modesto, CA). Processing of the data were performed using monoisotope peaks.

\section{RESULTS AND DISCUSSION}

The mass spectra of the poly(MMA-co-tBMA)s were predicted to be complicated, due to their random comonomer distribution combined with a variety of end-group combinations. Prior to the MALDI analyses, therefore, a mass list of possible components was computed to assist peak assignments. Figure 1 shows the possible chemical structures of the copolymer chains with different end-group combinations in the poly(MMA-co-tBMA)s. There are two types of possible initial ends (AVN initiation and EL initiation chains), because radical copolymerization was carried out using AVN as an initiator in EL acting as a chain transfer agent. At terminal ends, due to radical disproportionation, pairs of unsaturated and saturated groups are formed. Saturated terminals will also be formed by chain transfer reaction. Recombination of two radical chains with an AVN initial end or an EL initial end will also occur. As a result of these reactions, it can be assumed that poly(MMA-cotBMA)s has seven different types of end-group combinations, as shown in Fig. 1.

Figure 2 shows mass spectra of M45, illustrating the broad-band mass spectrum and the expanded mass spectrum in the range of $\mathrm{m} / \mathrm{z} 1,200-1,250$. Table 2 is a partial mass list corresponding to the expanded mass range. In this list, the calculated mass values appear as $[\mathrm{M}+\mathrm{Na}]^{+}$ions of putative copolymer components, denoted by their copolymer compositions and the types of end-group combination, together with the observed mass values and errors. Minimum mass differences in the theoretical mass of the isobaric components within poly(MMA-co-tBMA)s are about $0.036 \mathrm{Da}$, which is attributed to the differences in elemental compositions between $\mathrm{CO}$ and $\mathrm{C}_{2} \mathrm{H}_{4}$. The peak resolution of 40,000-60,000 (FWHM) could be achieved up to $\mathrm{m} / \mathrm{z}$ 3,000 , enabling the separation of a number of isobaric peaks having the mass differences in $0.036 \mathrm{Da}$, for example the isobaric peaks at $m / z 1218.714$ for $\operatorname{II}\left(\mathrm{M}_{8} \mathrm{~B}_{2}\right)$ and $m / z 1218.748$ for III $\left(\mathrm{M}_{4} \mathrm{~B}_{4}\right)$. Here, the adjacent peak at $m / z 1218.646$ could be assigned as the isotope peak of $\mathrm{I}\left(\mathrm{M}_{8} \mathrm{~B}_{2}\right)$. Among the 30 kinds of putative copolymer components in this mass range, the peaks of 19 components could be observed in M45, as labeled in the expanded mass spectrum of Fig. 2. Here, Roman numerals indicate the types of chain structure defined in Fig. 1, and $\left(\mathrm{M}_{m} \mathrm{~B}_{n}\right)$ indicates the comonomer composition of MMA (M) with $m$ units and tBMA (B) with $n$ units.

Figure 3 shows a comparison of the mass spectra of four poly(MMA-co-tBMA)s expanded in the range of $\mathrm{m} / \mathrm{z}$ $1,200-1,250$. To simplify this figure, the peak assignments are mainly limited to components with Type II chains.

Table 1. Preparation of poly(MMA-co-tBMA)s.

\begin{tabular}{|c|c|c|c|c|c|}
\hline \multirow{2}{*}{ Code } & \multicolumn{2}{|c|}{ Compositions of MMA (mol\%) } & \multicolumn{3}{|c|}{ Molecular weight parameters ${ }^{\mathrm{a}}$} \\
\hline & Feed & ${ }^{1} \mathrm{H}$ NMR & $M_{n}$ & $M_{w}$ & $M_{w} / M_{n}$ \\
\hline M12 & 12.0 & 12.3 & 3,470 & 6,300 & 1.82 \\
\hline M45 & 46.0 & 44.9 & 3,340 & 5,810 & 1.74 \\
\hline M64 & 66.0 & 64.0 & 3,330 & 5,390 & 1.62 \\
\hline M89 & 92.0 & 89.1 & 3,710 & 5,410 & 1.46 \\
\hline
\end{tabular}

${ }^{a}$ Determined by SEC. 


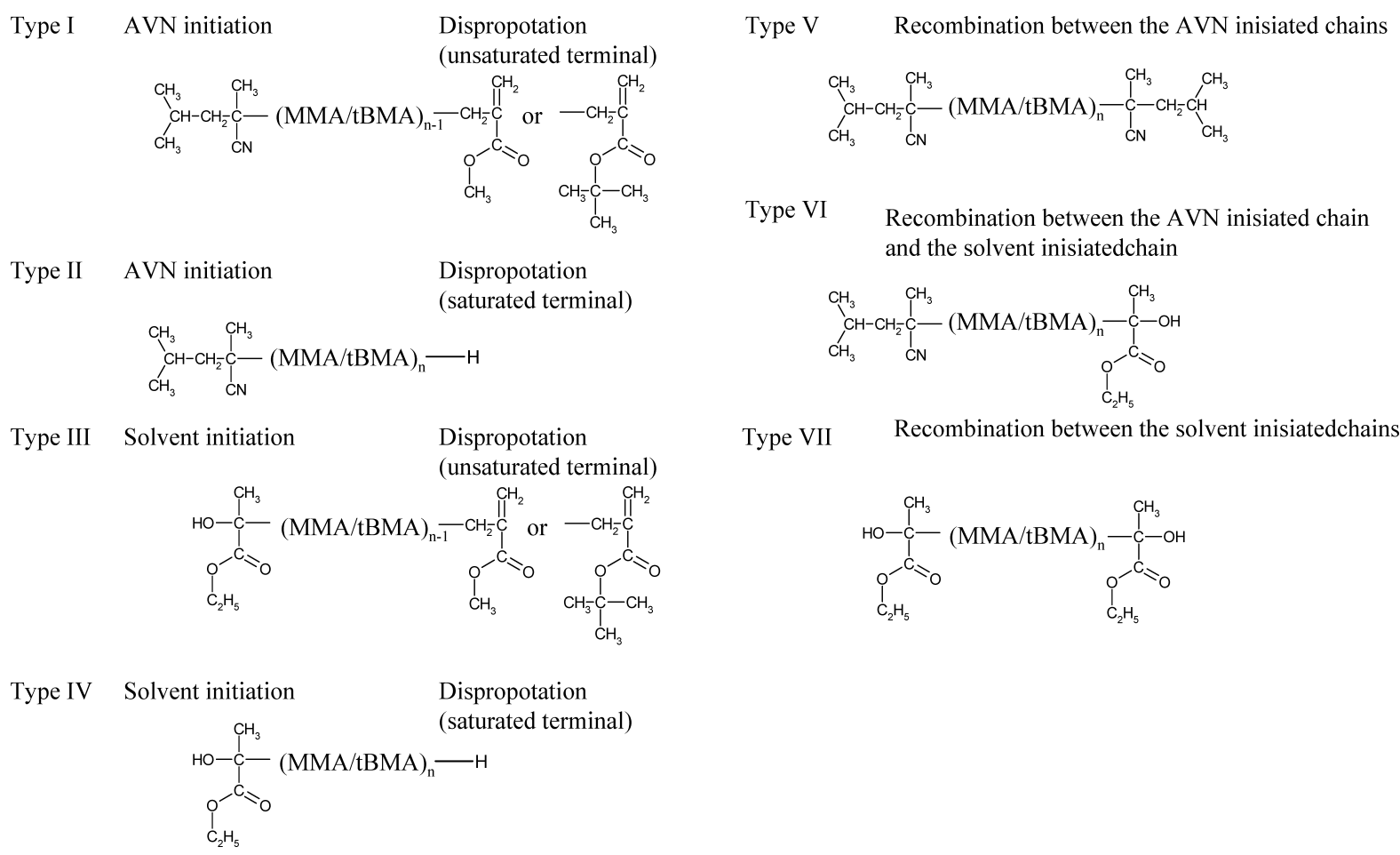

Fig. 1. Possible chemical structures of poly(MMA-co-tBMA)s.

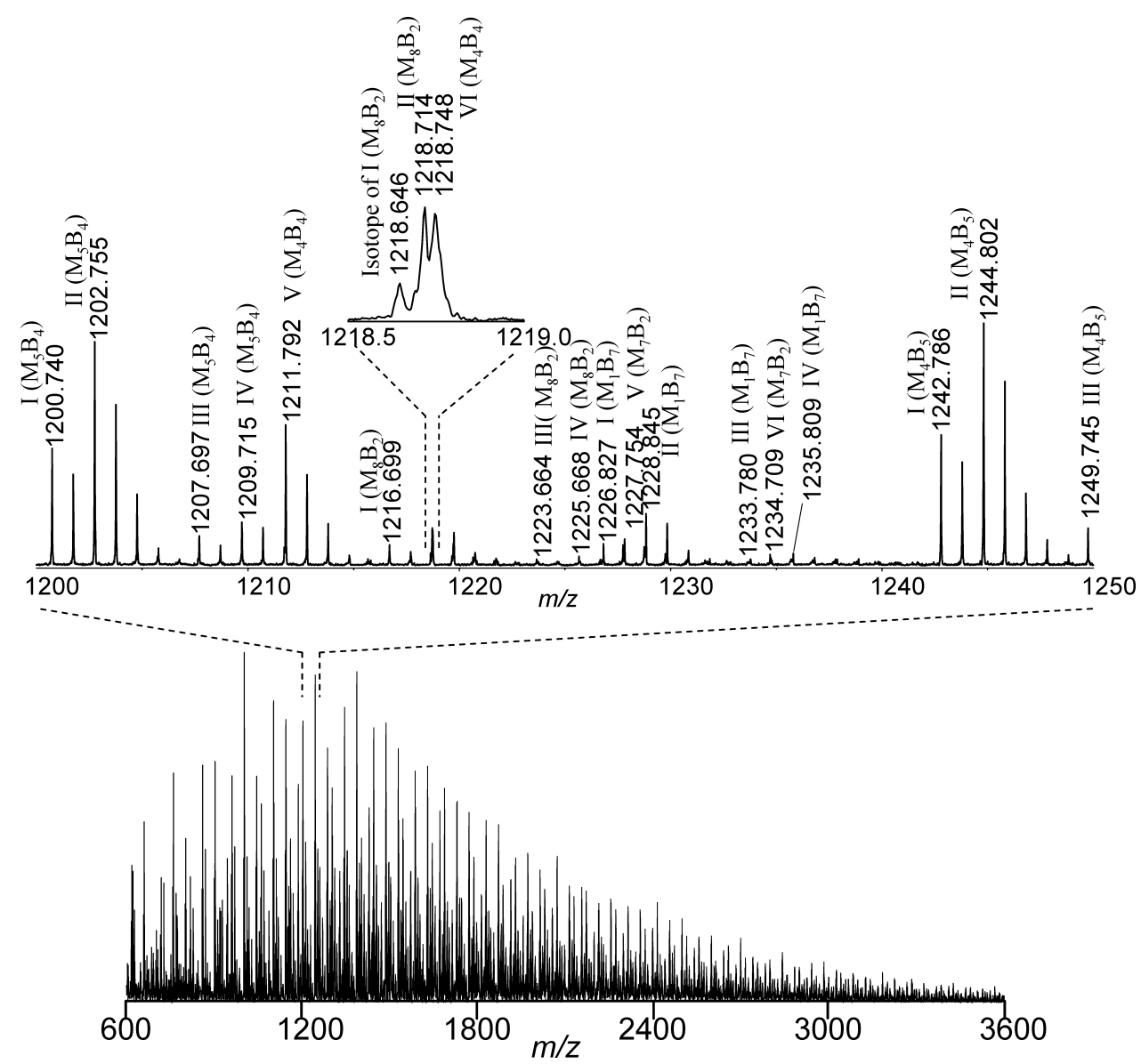

Fig. 2. Mass spectra of M45. Peak labels in the expanded mass spectra indicate the type of copolymer chain. Roman numerals indicate the structure types shown in Fig. 1 and $\left(\mathrm{M}_{m} \mathrm{~B}_{n}\right)$ indicates the comonomer composition of MMA (M) with $m$ units and tBMA (B) with $n$ units. 
Table 2. Candidate copolymers with a mass in the range of $m / z 1,200-1,250$ and assigned components of the M45 sample.

\begin{tabular}{|c|c|c|c|c|c|c|}
\hline \multicolumn{2}{|c|}{ Numbers of comonomer units } & \multirow{2}{*}{$\begin{array}{l}\text { Types of end-group } \\
\text { combinations }^{\mathrm{a}}\end{array}$} & \multicolumn{2}{|c|}{$m / z$ values $^{\mathrm{b}}$} & \multicolumn{2}{|c|}{ Errors } \\
\hline MMA & tBMA & & Calculated & Observed & in $\mathrm{Da}$ & in ppm \\
\hline 5 & 4 & $\mathrm{I}$ & 1200.739 & 1200.740 & 0.001 & 0.8 \\
\hline 5 & 4 & II & 1202.754 & 1202.755 & 0.001 & 0.8 \\
\hline 1 & 6 & VI & 1202.791 & nd & & \\
\hline 5 & 4 & III & 1207.697 & 1207.697 & 0.000 & 0.0 \\
\hline 5 & 4 & IV & 1209.712 & 1209.715 & 0.003 & 2.5 \\
\hline 1 & 6 & VII & 1209.749 & nd & & \\
\hline 4 & 4 & $\mathrm{~V}$ & 1211.791 & 1211.792 & 0.001 & 0.8 \\
\hline 8 & 2 & $\mathrm{I}$ & 1216.697 & 1216.699 & 0.002 & 1.6 \\
\hline 8 & 2 & II & 1218.713 & 1218.714 & 0.001 & 0.8 \\
\hline 4 & 4 & VI & 1218.749 & 1218.748 & -0.001 & -0.8 \\
\hline 8 & 2 & III & 1223.655 & 1223.664 & 0.009 & 7.4 \\
\hline 8 & 2 & IV & 1225.671 & 1225.668 & -0.003 & -2.4 \\
\hline 4 & 4 & VII & 1225.707 & nd & & \\
\hline 1 & 7 & I & 1226.827 & 1226.827 & 0.000 & 0.0 \\
\hline 7 & 2 & $\mathrm{~V}$ & 1227.749 & 1227.754 & 0.005 & 4.1 \\
\hline 1 & 7 & II & 1228.843 & 1228.845 & 0.002 & 1.6 \\
\hline 11 & 0 & $\mathrm{I}$ & 1232.656 & nd & & \\
\hline 1 & 7 & III & 1233.785 & 1233.780 & -0.005 & -4.1 \\
\hline 11 & 0 & II & 1234.671 & nd & & \\
\hline 7 & 2 & VI & 1234.708 & 1234.709 & 0.001 & 0.8 \\
\hline 1 & 7 & IV & 1235.801 & 1235.809 & 0.008 & 6.5 \\
\hline 0 & 7 & $\mathrm{~V}$ & 1237.879 & nd & & \\
\hline 11 & 0 & III & 1239.614 & nd & & \\
\hline 11 & 0 & IV & 1241.629 & nd & & \\
\hline 7 & 2 & VII & 1241.666 & nd & & \\
\hline 4 & 5 & I & 1242.786 & 1242.786 & 0.000 & 0.0 \\
\hline 10 & 0 & $\mathrm{~V}$ & 1243.708 & nd & & \\
\hline 4 & 5 & II & 1244.801 & 1244.802 & 0.001 & 0.8 \\
\hline 0 & 7 & VI & 1244.838 & nd & & \\
\hline 4 & 5 & III & 1249.744 & 1249.745 & 0.001 & 0.8 \\
\hline
\end{tabular}

a The chemical structures of each type of end-group combinations are shown in Fig. 1 above. ${ }^{\text {b }}$ nd means "not detected."

Related isobaric components are indicated in italic. In this mass range, five kinds of the Type II chains, i.e., $\mathrm{II}\left(\mathrm{M}_{5} \mathrm{~B}_{4}\right)$, $\mathrm{II}\left(\mathrm{M}_{8} \mathrm{~B}_{2}\right), \mathrm{II}\left(\mathrm{M}_{1} \mathrm{~B}_{7}\right), \mathrm{II}\left(\mathrm{M}_{11} \mathrm{~B}_{0}\right)$, and $\mathrm{II}\left(\mathrm{M}_{4} \mathrm{~B}_{5}\right)$, can be detected. As expected, with rising MMA composition of the samples, the relative peak intensities of the MMA-rich components become more intense. It should be noted that some of these peaks will overlap other isobaric components or isotopes. The high resolving power of spiral-TOFMS enabled us to separate most of these peaks. The right side of Fig. 3 was further expanded in the range of $\mathrm{m} / z$ 1226.5-1229.5. The monoisotope peaks of $\mathrm{I}\left(\mathrm{M}_{1} \mathrm{~B}_{7}\right)$ at $m / z 1226.827$ (theoretical) and $\mathrm{II}\left(\mathrm{M}_{1} \mathrm{~B}_{7}\right)$ at $\mathrm{m} / z 1228.843$ (theoretical) were clearly observed for higher tBMA component samples (M12 and $\mathrm{M} 45)$. For M45, the monoisotope peak of $\mathrm{V}\left(\mathrm{M}_{7} \mathrm{~B}_{2}\right)$ was observed at $m / z 1227.749$ (theoretical), close to the isotope peak of $\mathrm{I}\left(\mathrm{M}_{1} \mathrm{~B}_{7}\right)$ at $m / z 1227.830$ (theoretical). Increasing with the MMA composition, the relative peak intensities of $V\left(M_{7} B_{2}\right)$ were increased, whereas those of $\mathrm{I}\left(\mathrm{M}_{1} \mathrm{~B}_{7}\right)$ and $\mathrm{II}\left(\mathrm{M}_{1} \mathrm{~B}_{7}\right)$ relatively decreased. When conventional TOFMS instruments with lower resolving power are used, there is a risk of confusing the monoisotope peak of $\operatorname{II}\left(\mathrm{M}_{1} \mathrm{~B}_{7}\right)$ with the isotope peak of $\mathrm{V}\left(\mathrm{M}_{7} \mathrm{~B}_{2}\right)$. Isobaric peaks like this were distributed over the entire mass range. MALDI spiral-TOFMS was able to clearly distinguish these isobaric peaks and assign each peak to a unique component. Here, the monoisotope peaks of the Type II and Type IV chains overlapped with isotope peaks $(+2 \mathrm{Da})$ of Type I and Type III chains with the same copolymer composition and whose mass differences were only $0.01 \mathrm{Da}$, that could not be separated even by the spiralTOFMS equipment. The peak intensities of these components could be roughly corrected in consideration of isotope distribution. A set of molecular species and peak intensity were thus obtained for the entire mass spectrum, making it possible to perform compositional characterization.

An important consideration when determining copolymer composition is that the peak intensities in MALDI mass spectra do not always reflect the relative abundance of the species they represent. Two types of discrimination should be taken into consideration. One is mass discrimination, caused mainly by differences in ion formation and/or ion detection that depend on mass. ${ }^{18)}$ In general, higher mass components are difficult to observe when MALDI TOFMS is applied to polymers with a broad molecular weight distribution (typically, a polydispersity index, PDI, that exceeds 1.1). In fact, the observed peak distributions of the samples were shifted to the lower mass region than average molecular weight as determined by SEC, as shown in Fig. 2. The other type of discrimination is compositional bias, caused by differences in ionization efficiency that are a function of chemical structure. The influences of these two types of discrimination on the compositional characterization of the poly(MMA-co-tBMA)s were examined.

Figure 4 shows the bivariate distributions of copolymer composition of four poly(MMA-co-tBMA)s based on their observed peak intensities. Although the mass discrimination effect influenced the observed molecular weight distribution, every bivariate distribution stretches in the direction of average copolymer composition, which is the typical topology of a random copolymer. ${ }^{8)}$ No "ghost" distribution appears beyond the reasonable range, suggesting suitable peak assignments by MALDI spiral-TOFMS. Table 3 summarizes the average copolymer compositions by MALDI 


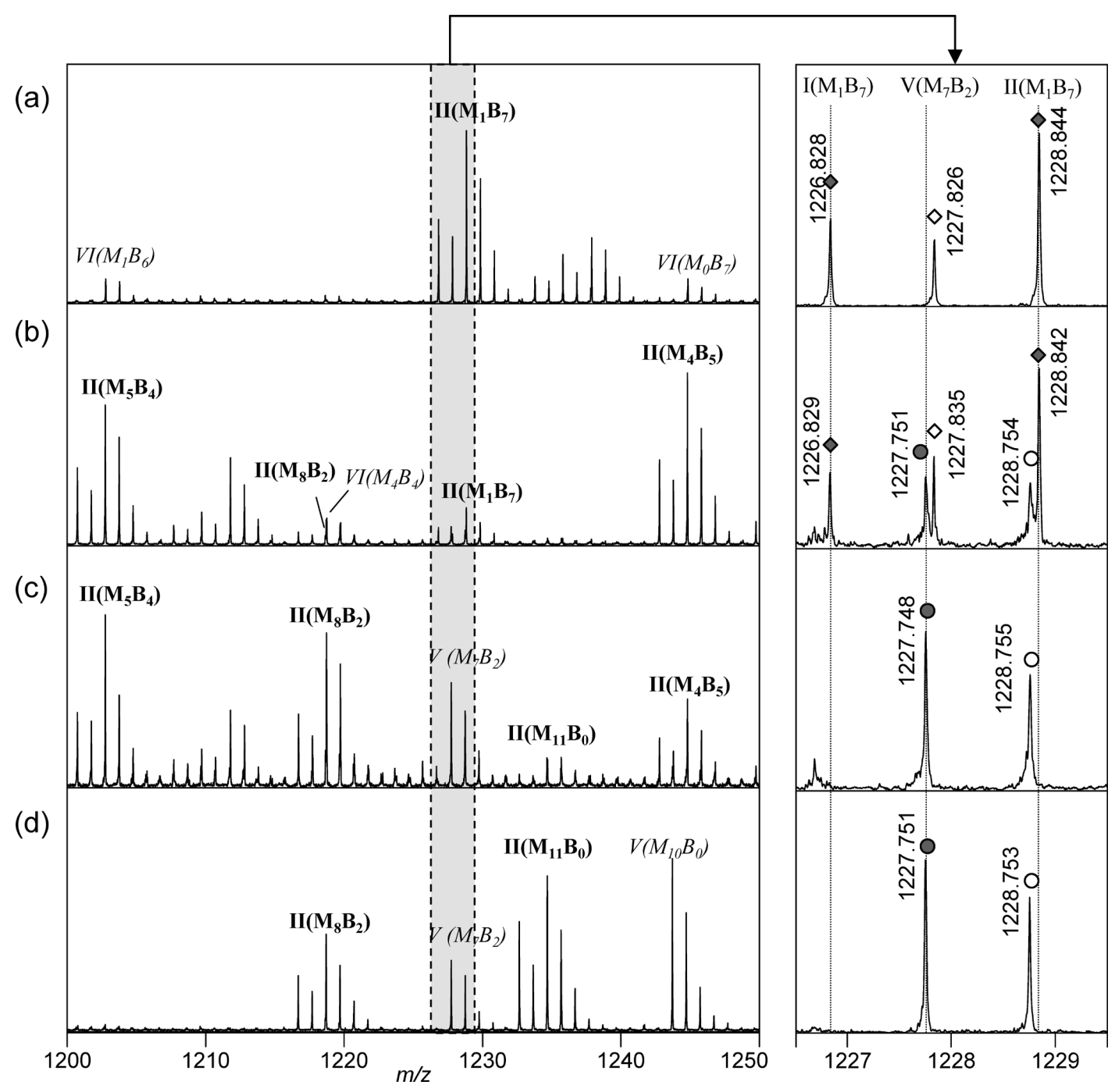

Fig. 3. Comparison of the mass spectra of (a) M12, (b) M45, (c) M64, and (d) M89 in the range of $m / z 1200-1250$ (left) and $m / z 1226.5-1229.5$ (right). The peaks on the left were mainly assigned to Type II chains (bold) together with related isobaric components (italic). Filled marks on the right correspond to monoisotope peaks and open marks correspond to isotope peaks.

spiral-TOFMS together with the ${ }^{1} \mathrm{H}$ NMR data. Because the copolymer compositions of poly(MMA-co-tBMA)s produced under high conversion conditions varied slightly, depending on the chain length up to 15 -mer, copolymer compositions were also obtained by averaging between 15-mer and 20-mer. Average copolymer compositions obtained by MALDI spiral-TOFMS were slightly higher than those obtained by ${ }^{1} \mathrm{H}$ NMR. A similar pattern has been reported by Suddaby et al. ${ }^{19)}$ for copolymers of MMA and $n$ butyl methacrylate (BMA) produced by free radical catalytic chain transfer polymerization, probably because ionization is more favored by MMA units than the more hydrophobic BMA units.

The influence of compositional bias was further evaluated using the chain statistical model. It has been reported that the copolymerization of methacrylates generally follows the terminal model. ${ }^{20)}$ However, since the monomer reactivity ratio for MMA/tBMA copolymerization has been reported as $r_{\mathrm{MMA}}=0.81 \pm 0.06$ and $r_{\mathrm{tBMA}}=1.26 \pm 0.03$, calculated using the Kelen-Tüdõs method, ${ }^{21)}$ if we assume random copolymerization, the ideal compositional distributions can be calculated according to Bernoullian chain statistics (Eq. (1)),

$$
\mathrm{I}\left(\mathrm{MMA}_{m}, \mathrm{tBMA}_{n}\right)=\frac{(m+n) !}{m ! n !}[\mathrm{MMA}]^{m}[\mathrm{tBMA}]^{n}
$$

where $\left.\mathrm{I}_{\left(\mathrm{MMA}_{m}, \mathrm{BMA}\right.}\right)$ is the composition of the copolymer chain composed of $m$ units of MMA and $n$ units of tBMA, and [MMA] and [tBMA] are the feed concentrations of each comonomer. Figure 5 shows a comparison of compositional distributions observed by MALDI spiral-TOFMS and the calculated distributions for 10-mer of each sample. The observed distributions were very similar to those calculated using Bernoullian chain statistics. These results suggest that the influence of compositional bias will be within the acceptable range for investigating copolymerization behavior.

The compositional distribution of end-group combinations was then evaluated. Figure 6 shows the relative peak intensities of each type of M45. In general, free radical polymerization mainly produces chains whose terminus are an initiator residue end and an unsaturated or saturated end, corresponding to Type I and Type II chains. An excess number of Type II chains having a saturated end will be caused by the chain transfer reaction in the presence of EL. The resultant EL radical will readily re-initiate a new propagation chain, ${ }^{22}$ forming Type III and Type IV chains by radical 

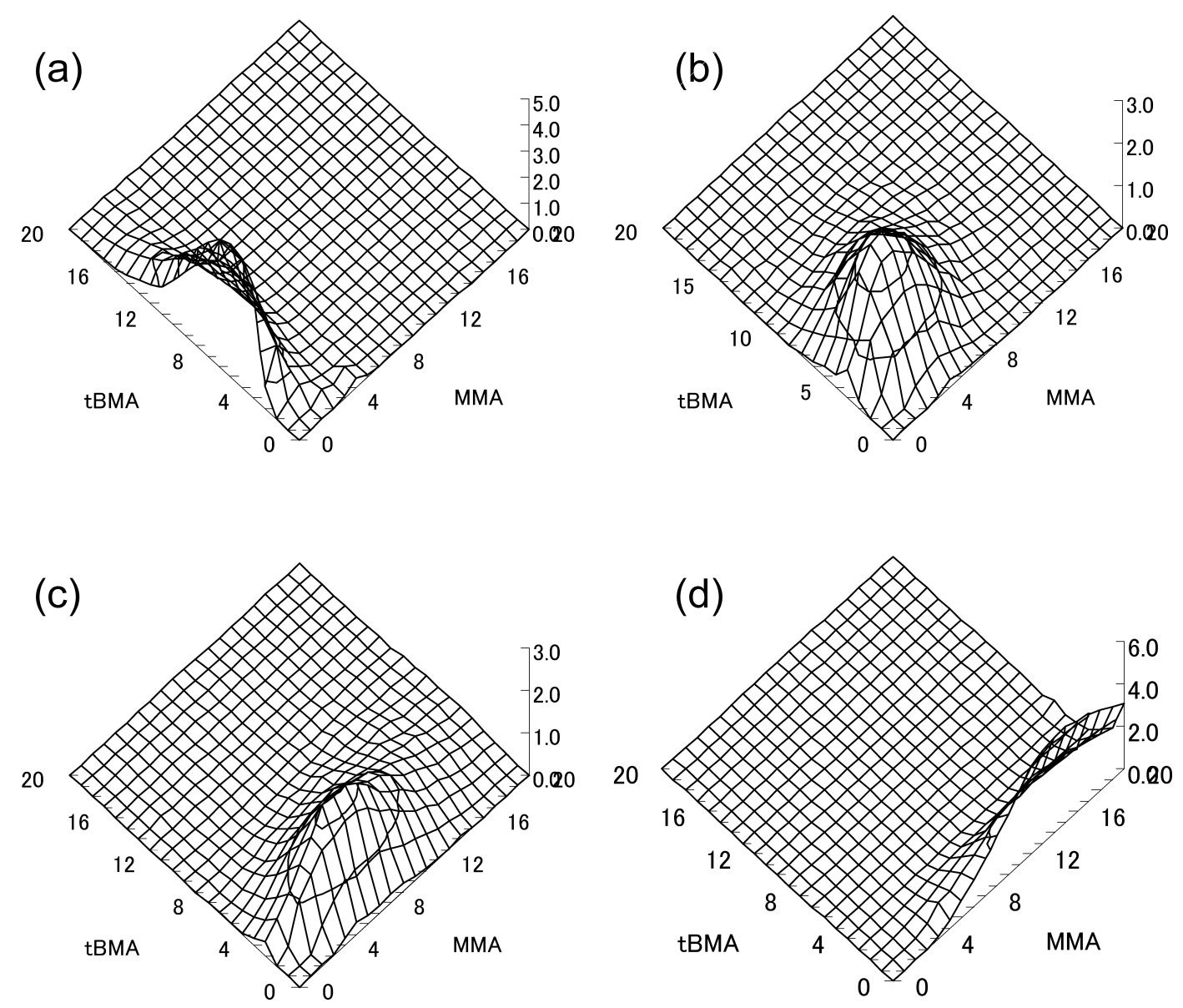

Fig. 4. Bivariate distributions of the copolymer composition of (a) M12, (b) M45, (c) M64, and (d) M89.

Table 3. Average MMA compositions of poly(MMA-co-tBMA)s.

\begin{tabular}{cccc}
\hline & \multicolumn{3}{c}{ MMA composition (mol\%) } \\
\cline { 2 - 4 } Code & & \multicolumn{2}{c}{ MALDI spiral-TOFMS } \\
\cline { 3 - 4 } & ${ }^{1}$ H NMR & $\begin{array}{c}\text { Whole mass } \\
\text { spectra }\end{array}$ & $\begin{array}{c}\text { Average between } \\
15-\text { mer and 20- } \\
\text { mer }\end{array}$ \\
& & $14.3(0.8)$ & $13.7(0.4)$ \\
M12 & 12.3 & $47.7(0.4)$ & $49.0(0.6)$ \\
M45 & 44.9 & $66.9(0.8)$ & $68.6(0.5)$ \\
M64 & 64.0 & $94.2(0.6)$ & $95.4(0.3)$ \\
M89 & 89.1 & &
\end{tabular}

${ }^{a}$ Data are the average for three different mass spectra. Figures in parentheses are the standard deviations.

disproportionation, or Type VI chains by recombination with the radicals of AVN-initiated chains. An excess number of Type IV chains over Type III chains is likely to be for the same reason as applies during the formation of Type II chains. A higher concentration of AVN initiator used in this study ( $5 \mathrm{~mol} \%$ of the monomer mixture) resulted in a moderate number of Type $\mathrm{V}$ chains formed by recombination. There were no obvious peaks representing Type VII chains formed by recombination with the radicals of the EL-initiated chains, probably due to the relatively lower concentration of EL-initiated chains. The compositional distribution of end-group combinations characterized by MALDI spiralTOFMS was therefore reasonable.

Because the chain reaction constant differs from comonomer species, the composition of the EL end will vary

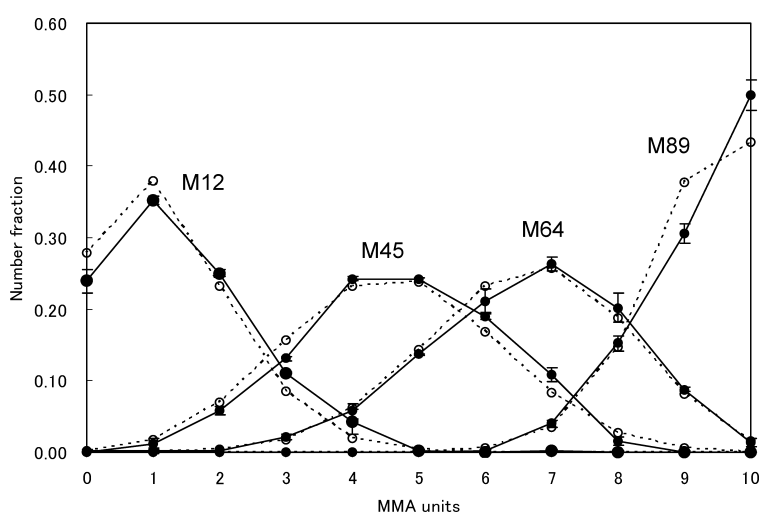

Fig. 5. Compositional distributions of 10-mers of four poly(MMAco-tBMA)s as obtained by MALDI spiral-TOFMS (O) and calculated using the Bernoullian statistical model $(\bigcirc)$. Error bars are given as standard deviation for three measurements.

according to the copolymer's composition. The relative proportion of EL-initiated chains $\left(R_{\mathrm{EL}}\right)$ was evaluated using Eq. (2) below,

$$
R_{\mathrm{EL}}=\frac{\sum P_{\text {typeIII }}+\sum P_{\text {typeIV }}}{\sum P_{\text {typeI }}+\sum P_{\text {typeII }}}
$$

where $P_{\text {Type } x}$ are the peak intensities of the Type $x$ chains ( $x=\mathrm{I}-\mathrm{IV})$. Figure 7 plots the obtained $R_{\mathrm{EL}}$ values against the MMA composition. The $R_{\mathrm{EL}}$ values considerably decreased on increasing the MMA composition, suggesting that chain 


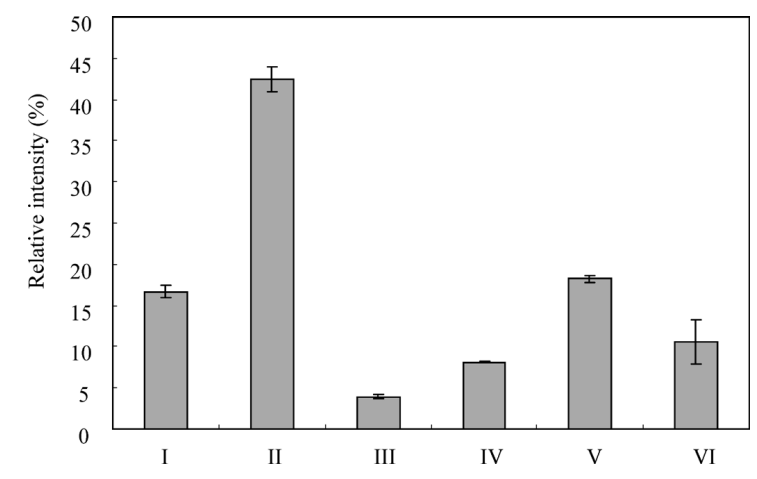

Fig. 6. Compositional distributions of each type chain of M45. Error bars are given as standard deviation for three measurements.

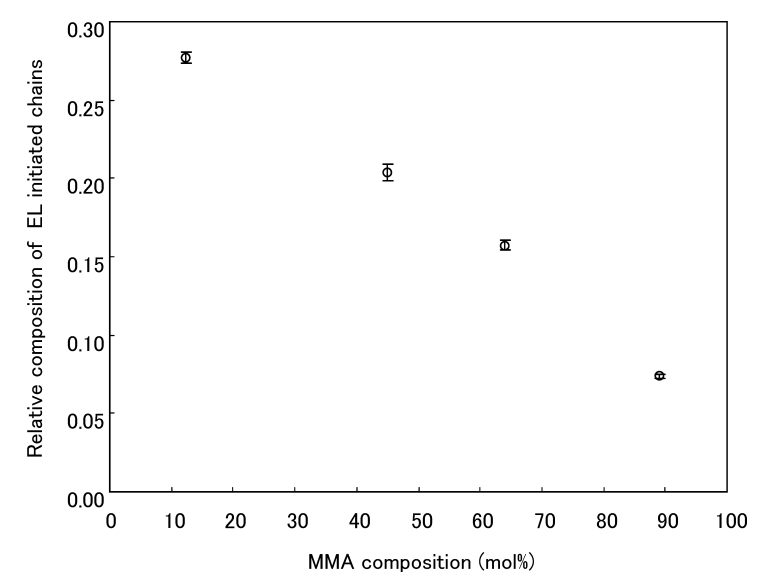

Fig. 7. Relationship between the relative compositions of EL-initiated chains and copolymer composition. Error bars are given as standard deviation for three measurements.

transfer from propagating MMA radicals to the EL molecules is an unfavorable reaction.

\section{CONCLUSION}

As demonstrated above, MALDI spiral-TOFMS made it possible to perform compositional characterization of poly(MMA-co-tBMA)s with a complicated compositional distribution resulting from various types of end-group combinations. Although the characterization of end-group combinations is a specific feature of MALDI analyses, the inadequacy of the resolving power of conventional TOFMS has impeded progress in copolymer characterization by MALDI analysis. In this study, six types of end-group combinations were identified within the high-resolution mass spectra, allowing the compositional distribution to be evaluated. The results obtained will be useful for further investigations of the states of chain transfer reactions and recombinations in free radical polymerization. In conclusion, MALDI spiralTOFMS has potential as a powerful tool for the structural and compositional characterization of complicated copolymers.

\section{REFERENCES}

1) G. Montaudo, F. Samperi, M. S. Montaudo. Characterization of synthetic polymers by MALDI-MS. Prog. Polym. Sci. 31: 277-357, 2006.
2) M. S. Montaudo. Mass spectra of copolymers. Mass Spectrom. Rev. 21: 108-144, 2002.

3) P. Terrier, W. Buchmann, G. Cheguillaume, B. Desmazières, J. Tortajada. Analysis of poly(oxyethylene) and poly(oxypropylene) triblock copolymers by MALDI-TOF mass spectrometry. Anal. Chem. 77: 3292-3300, 2005.

4) G. Adamus, P. Rizzarelli, M. S. Montaudo, M. Kowalczuk, G. Montaudo. Matrix-assisted laser desorption/ionization time-offlight mass spectrometry with size-exclusion chromatographic fractionation for structural characterization of synthetic aliphatic copolyesters. Rapid Commun. Mass Spectrom. 20: 804-814, 2006.

5) R. Alicata, T. Barbuzzi, M. Giuffrida, A. Ballistreri. Characterization of poly[(R)-3-hydroxybutyrate-co-epsilon-caprolactone] copolymers by matrix-assisted laser desorption/ionization timeof-flight and electrospray ionization mass spectrometry. Rapid Commun. Mass Spectrom. 20: 568-576, 2006.

6) F. Samperi, R. Mendichi, L. Sartore, M. Penco, C. Puglisi. Full characterization of a multiblock copolymer based on poly $(2,6$ dimethyl-1,4-phenylene oxide) and poly(bisphenol-A carbonate). Macromolecules 39: 9223-9233, 2006.

7) S. Huijser, B. B. P. Staal, J. Huang, R. Duchateau, C. E. Koning. Topology characterization by MALDI-ToF-MS of enzymatically synthesized poly(lactide-co-glycolide). Biomacromolecules 7: 2465-2469, 2006.

8) S. Huijser, B. B. P. Staal, J. Huang, R. Duchateau, C. E. Koning. Chemical composition and topology of poly(lactide-co-glycolide) revealed by pushing MALDI-TOF MS to its limit. Angew. Chem. Int. Ed. Engl. 45: 4104-4108, 2006.

9) G. J. van Rooij, M. C. Duursma, C. G. de Koster, R. M. A. Heeren, J. J. Boon, P. J. W. Schuyl, E. R. E. van der Hage. Determination of block length distributions of poly(oxypropylene) and poly(oxyethylene) block copolymers by MALDI-FTICR mass spectrometry. Anal. Chem. 70: 843-850, 1998.

10) T. H. Mize, W. J. Simonsick Jr., I. J. Amster. Characterization of polyesters by matrix-assisted laser desorption/ionization and Fourier transform mass spectrometry. Eur. J. Mass Spectrom. (Chichester, Eng.) 9: 473-486, 2003.

11) F. J. Cox, K. N. Qian, A. O. Patil, M. V. Johnston. Microstructure and composition of ethylene-carbon monoxide copolymers by matrix-assisted laser desorption/ionization mass spectrometry. Macromolecules 36: 8544-8550, 2003.

12) S. M. Miladinovic, C. J. Kaeser, M. M. Knust, C. L. Wilkins. Tandem Fourier transform mass spectrometry of block and random copolymers. Int. J. Mass Spectrom. 301: 184-194, 2011.

13) T. Satoh, H. Tsuno, M. Iwanaga, Y. Kammei. The design and characteristic features of a new time-of-flight mass spectrometer with a spiral ion trajectory. J. Am. Soc. Mass Spectrom. 16: 1969-1975, 2005.

14) T. Satoh, T. Sato, J. Tamura. Development of a high-performance MALDI-TOF mass spectrometer utilizing a spiral ion trajectory. J. Am. Soc. Mass Spectrom. 18: 1318-1323, 2007.

15) L. Ulmer, J. Mattay, H. G. Torres-Garcia, H. Luftmann. Letter: The use of 2-[(2E)-3-(4-tert-butylphenyl)-2-methylprop-2-enylidene]malononitrile as a matrix for matrix-assisted laser desorption/ionization mass spectrometry. Eur. J. Mass Spectrom. (Chichester, Eng.) 6: 49-52, 2000.

16) M. F. Wyatt, B. K. Stein, A. G. Brenton. Characterization of various analytes using matrix-assisted laser desorption/ionization time-of-flight mass spectrometry and 2-[(2E)-3-(4-tertbutylphenyl)-2-methylprop-2-enylidene]malononitrile matrix. Anal. Chem. 78: 199-206, 2006.

17) J. De Winter, G. Deshayes, F. Boon, O. Coulembier, P. Dubois, P. Gerbaux. MALDI-ToF analysis of polythiophene: use of trans-2-[3-(4-t-butyl-phenyl)-2-methyl-2-propenylidene]malononitrile-DCTB-as matrix. J. Mass Spectrom. 46: 237-246, 2011.

18) G. Montaudo, M. S. Montaudo, C. Puglisi, F. Samperi. Characterization of polymers by matrix-assisted laser-desorption 
ionization time-of-flight mass-spectrometry-molecular-weight estimates in samples of varying polydispersity. Rapid Commun. Mass Spectrom. 9: 453-460, 1995.

19) K. G. Suddaby, K. H. Hunt, D. M. Haddleton. MALDI-TOF mass spectrometry in the study of statistical copolymerizations and its application in examining the free radical copolymerization of methyl methacrylate and $n$-butyl methacrylate. Macromolecules 29: 8642-8649, 1996

20) H. Yuki, Y. Okamoto, Y. Shimada, K. Ohta, K. Hatada. Microstructure of the copolymers of methyl-methacrylate with other methacrylates obtained by radical and anionic copolymerizations in tetrahydrofuran. J., Polym. Sci., Polym. Chem. 17: 1215-1225, 1979.

21) H. Momose, T. Maeda, K. Hattori, T. Hirano, K. Ute. Statistical determination of chemical composition and monomer sequence distribution of poly(methyl methacrylate-co-tert-butyl methacrylate)s by multivariate analysis of ${ }^{13} \mathrm{C}$ NMR spectra. Polym. J. 44: 808-814, 2012.

22) E. Ranucci, L. Macchi, R. Annunziata, P. Ferruti, F. Chiellini. End-functionalised 1-vinyl-2-pyrrolidinone oligomers bearing lactate functions at one end. Macromol. Biosci. 4: 706-713, 2004. 